\title{
Quantifying the causal impact of funding bedside antigen testing on the incidence of respiratory syncytial virus infection in Japan: a difference-in-differences study
}

\author{
Sung-mok Jung ${ }^{1,2,3} \wedge$, Hyojung Lee ${ }^{1,2,4}$, Yichi Yang ${ }^{1}$, Hiroshi Nishiura ${ }^{1,2,3} \wedge$ \\ ${ }^{1}$ Graduate School of Medicine, Hokkaido University, Sapporo-shi, Hokkaido, Japan; ${ }^{2}$ CREST, Japan Science and Technology Agency, Saitama, \\ Japan; ${ }^{3}$ Kyoto University School of Public Health, Kyoto, Japan; ${ }^{4}$ National Institute for Mathematical Sciences, Daejeon, Korea \\ Contributions: (I) Conception and design: H Nishiura, SM Jung, H Lee; (II) Administrative support: None; (III) Provision of study materials or \\ patients: None; (IV) Collection and assembly of data: SM Jung, H Lee, Y Yang; (V) Data analysis and interpretation: SM Jung, H Lee, H Nishiura; (VI) \\ Manuscript writing: All authors; (VII) Final approval of manuscript: All authors. \\ Correspondence to: Hiroshi Nishiura. Kyoto University School of Public Health, Yoshidakonoecho, Sakyo-ku, Kyoto 606-8503, Japan. \\ Email: nishiura.hiroshi.5r@kyoto-u.ac.jp.
}

\begin{abstract}
Background: To estimate the causal impact of a change in the national health insurance policy to cover the cost of respiratory syncytial virus (RSV) antigen testing on the incidence of RSV infection by age-groups, we analyzed the sentinel datasets of RSV infection in Japan from 2009-2017.

Methods: The causal effect of introducing RSV antigen testing on increasing the reported incidence of RSV infection was quantified using a quasi-experimental difference-in-differences (DID) design and influenza as a control group.

Results: Examining sentinel and virus surveillance datasets, only clinically mild cases have selectively increased from 2012/13. The median estimated incidences of influenza and RSV infection among children aged 0-4 years, the age group that dominates cases of both diseases, were estimated at 5,607 and 1,761 per 100,000 individuals, respectively. Exploring sentinel datasets, only the incidence of RSV infection abruptly increased from 2012/13. Using an age-dependent model, the estimated causal effect on the increase in RSV annual incidence was greatest among children aged 5-9 years, with an estimated additional 1,912 cases per 100,000 individuals (95\% CI: 418-3,406).

Conclusions: Owing to financial support of bedside RSV antigen testing from 2012/13, the incidence of RSV infection has been elevated. The recent increasing trend in RSV infection incidence should not be directly perceived as an increase in natural infections with RSV.
\end{abstract}

Keywords: Respiratory syncytial virus (RSV); bedside antigen testing; influenza; difference-in-differences (DID); Japan

Submitted Jan 08, 2020. Accepted for publication Aug 16, 2020.

doi: $10.21037 /$ atm-20-423

View this article at: http://dx.doi.org/10.21037/atm-20-423

\section{Introduction}

Respiratory syncytial virus (RSV) is the leading cause of acute lower respiratory infections among infants as well as an important cause of infant deaths in developing countries (i.e., mortality among those aged $<5$ years) (1). Clinically, RSV infection among children who are $<24$ months of age initially involves cough, rhinorrhea, and fever, with severe infections manifesting as bronchiolitis, pneumonia, and cardiovascular disorders (1-3). RSV transmissibility

\footnotetext{
^ Sung-mok Jung, ORCID: 0000-0002-0787-4515; Hiroshi Nishiura, ORCID: 0000-0003-0941-8537.
} 
is considered to be substantial, and lifelong immunity is not elicited following the primary infection, permitting infections among the majority of infants before their third year of life (4-7). Globally, RSV infection induced 66,000199,000 deaths in 2005 (8). Moreover, with an apparent seasonality, the annual cycle of RSV infection is observed from autumn to spring, with variable timing of the epidemic peak by year and region $(9,10)$. In tropical and subtropical areas, such as Hong Kong, RSV infection peaks with high temperature during the rainy season (11-13). Currently, vaccines to prevent RSV infection have yet to be put into practice, but palivizumab, a monoclonal antibody that offers passive immunity against RSV, can be administered to highrisk patients, such as premature babies with chronic lung disease or congenital heart disease (14-16).

In Japan, RSV infection is designated as a category $\mathrm{V}$ infectious disease by the infectious disease law $(17,18)$. Sentinel surveillance for RSV has been practiced since 2003, monitoring the weekly reports of RSV infections that were diagnosed via laboratory-confirmation (e.g., RT-PCR) and rapid diagnostic testing (RDT) kits (17). The RDT is known to be cost-effective (19), reducing the lengths of hospitalization and antibiotic treatment, with the estimated sensitivity and specificity at $81.8 \%$ and $100.0 \%$, respectively (20). Annually, there are RSV reports from about 3,000 sentinel pediatric hospitals and clinics across 47 prefectures in Japan $(21,22)$. The incidence of RSV infection displays a dramatic increase that began in the 2010/11 season. Such an abrupt increase should ideally be interpreted in an explicit manner, distinguishing the true increase in the incidence from an artificial increase in disease reports due to policy change, so that the importance of RSV epidemics can be quantified appropriately (21). The Japanese national health insurance originally approved covering the cost of RSV antigen testing among only hospitalized patients, but, since 17 October 2011, the national medical insurance extended its coverage to all infants under 12 months of age as well as to outpatients for whom palivizumab is indicated $(18,21)$. Therefore, it is crucial to understand (I) if there was any visible impact of the insurance policy change on the epidemiology of RSV in Japan and (II) how much the introduction of RDT has influenced the increase in reported RSV incidence.

A difference-in-differences (DID) study design, which is a quasi-experimental epidemiological study design, is a useful tool for estimating the time-dependent causal effect. DID design purifies the causal effect from time-series data, adjusting for any changes that occurred for both exposed and control group. Because the causal effect is estimated by comparing the changes in outcomes over time between exposed and control groups, selecting control groups that have a shared parallel trend with the exposed group during the pre-intervention period is crucial $(23,24)$. DID is recognized as a useful tool for evaluating the effect of health care policy changes (25).

While the incidence of RSV infection before 2012/13 has been linked to an increase in the number of reporting sentinel sites elsewhere (21), the recent increase in the incidence from 2012/13 season is one of the major public health concerns in Japan. If this increase has not driven by the introduction of funded RSV antigen testing, the underlying biological reasons should be explored, and in fact, the notification is known to have increased remarkably from sentinel clinics rather than from hospitals (21). Therefore, quantifying the causal effect of this changes in the national health insurance on the incidence of RSV infection is deemed important for verifying the phenomenon. The observation of a different trend in the incidences of influenza (the control group for this study) and RSV infection since the 2012/13 season would offer an important avenue by which to quantify the causal impact of introducing a funded RDT kit on the observed increase in the diagnosis of RSV infections, e.g., the increased diagnoses made using bedside antigen testing may encompass a broader spectrum of patients. The purpose of the present study was to estimate the impact of a change in the national health insurance policy on the reporting of RSV infection, analyzing the sentinel surveillance data of influenza and RSV infections in Japan and applying a DID study design to this context. We present the following article in accordance with the MDAR checklist (available at http://dx.doi.org/10.21037/atm-20-423).

\section{Methods}

\section{Epidemiological data}

The present study was conducted using publicly available datasets of the number of influenza cases and RSV infections, as reported by sentinel surveillance institutes and clinics across Japan from 2008-2017. The sentinel surveillance of RSV infection is based on samples of 3,000 pediatric sentinel medical facilities, regardless of hospitalization (i.e., including primary care visit, emergency care and inpatient data), while the numbers of influenza cases are notified from 5,000 adult and pediatric sentinel 
facilities across Japan (26). RSV infection is reported (I) when a patient exhibits any clinical characteristics that lead to suspect RSV infection, typically starting with upper respiratory symptoms (e.g., nasal discharge and coughing), followed by lower respiratory symptoms, and (II) when the virus is detected by means of RT-PCR or a RDT kit, based on immunochromatography, from nasal swabs, or a serological diagnosis is made using neutralization or complement fixation methods $(27,28)$. The reporting of influenza requires the patient to have (I) all four clinical characteristics, including sudden onset, high grade fever, upper respiratory symptoms, and general fatigue, and/ or (II) positive test results from RDT of influenza antigen using nasal or pharyngeal swabs (29). Notably, due to the widespread use of RDT for influenza in Japan, the majority of reported influenza cases are considered to represent actual influenza cases, thus mirroring the entire picture of influenza epidemiology (30).

Prefecture-dependent and age-specific incidence per 100,000 individuals were retrieved from the Infectious Disease Weekly Report provided by the National Institute of Infectious Diseases, Japan (26). Moreover, we examined prefecture- and age-specific population estimates from 2009-2017, retrieving them from the Statistics Bureau of Japan (31). RSV infection is a major cause of respiratory illness among young children, and thus, the reported cases have mostly been only in children. The age-specific data of both influenza and RSV infection offered us the number of reported cases for the entire population, split into five age groups with an emphasis on children, i.e., our subject ages were grouped as $0-4,5-9,10-14,15-19$, and $\geq 20$ years. Note that prefecture data (i.e., the number of reported cases of RSV infection and ILI per 100,000 individuals residing in each prefecture in Japan) provide us with the estimated impact of the national insurance coverage of the total population.

\section{Descriptive and trend analyses}

We first conducted a descriptive analysis of the multitudes of datasets. In addition to sentinel surveillance data, we analyzed the virus surveillance data, namely, weekly number of reported RSV detections from 2008-2017. The virus surveillance is laboratory-based pathogen surveillance, collecting laboratory testing results from prefectural public health institutes across Japan. In general, only severe fraction of cases are tested in those institutes, and virus surveillance mirrors the trend of severe RSV infections. Moreover, we have explored RSV cases by clinical prognosis (i.e., recovery or death) and also by differential type of medical attendance. Using fatal RSV cases that were derived from on the causeof-death statistics, we estimated the case fatality risk (CFR) every year. RSV notifications were classified into those from hospitals and clinics, and those from the latter are considered as clinically mild cases. Retrieving the corresponding published numbers (21) and calculating the proportion of notifications from clinics out of the total notifications from 2008-2015, we examined if the proportion of clinically severe RSV infections varied over time.

\section{Quasi-experimental analysis}

Subsequently, considering advantages of the DID design (e.g., accounting for changes in time trend and controlling for measured and a part of unmeasured confounding), a DID design was employed to estimate the causal effect on the RSV incidence of a change in the national health insurance coverage to newly cover the cost of RSV antigen detection testing among outpatients in pediatric settings. We visually assessed the parallel trends of incidence shared by influenza and RSV infection, verifying that these two common infectious diseases among children perhaps shared a comparable trend of infection, severity, and diagnosis prior to the change in bedside antigen testing for RSV that occurred in the 2012/13 season. Influenza was specifically selected as a control group in this study because of the absence of breakthroughs in prevention and treatment and the parallel features of incidence for RSV infection and influenza, which is a major assumption to ensure the validity of DID design. Moreover, both diseases share similar epidemiological characteristics (i.e., respiratory tract infection with seasonality and age distribution in the number of cases) and identical notification category (i.e., classified as type $\mathrm{V}$ infectious diseases in Japan, which need to be notified via sentinel surveillance systems, particularly from pediatric sentinel institutes and clinics). The change in the incidence of influenza from the pre-intervention period to the post-intervention period (i.e., 2012/13-2016/17) was assumed to be a valid counterfactual for the change that would have occurred in the incidence of RSV infection if not for the change in national health insurance coverage for RSV antigen testing.

To test the validity of the assumption of linear trend prior to the change in health insurance coverage, the difference in the slope of the linear trend during the pre-intervention period (i.e., 2008/09-2011/12) between influenza and 
RSV infection was estimated by age group, employing a linear regression model. The methodology taken to test for differences in time trend are detailed in Supplementary file (Appendix 1). Using two different types of datasets, i.e., prefecture- and age-specific data, two different DID models were independently applied. Age-specific incidence was examined, because both infectious diseases are dominated by cases of children aged from 0-4 years old (especially, during the first 2 years of life for RSV infection).

We used $t=2008 / 09,2009 / 10,2010 / 11,2011 / 12$, $2012 / 13,2013 / 14,2014 / 15,2015 / 16$, and $2016 / 17$ to indicate the epidemic season and $\mathrm{g}=1, \ldots, \mathrm{G}$ to index the geographic locations of the 47 prefectures in Japan (i.e., G =47). Each season represents 1-year starting from week 27 of the first listed year to week 26 of the following year.

We set $\widehat{Y_{g t}}$ as representing the outcome, i.e., the incidence of influenza or RSV in prefecture $g$ in year $t$. For the analysis of prefectural data, the expected value of the incidence was calculated as:

$$
\widehat{Y_{g t}}=\hat{\alpha}+\hat{\beta}\left(R S V_{g} T_{t}\right)+\hat{\gamma} R S V_{g}+\hat{\delta} T_{t}
$$

where $\hat{\alpha}$ represents the baseline incidence for both infections, $\hat{\beta}$ is the causal effect of the change in national health insurance on RSV antigen detection testing, $\hat{\gamma}$ is the difference in baseline incidence for RSV infection compared to influenza, and $\hat{\delta}$ is the time-dependent effect on infections of RSV and of the control group disease, influenza. $R S V_{g}$ and $T_{t}$ are dichotomous dummy variables, defined as:

$$
R S V_{g}=\left\{\begin{array}{c}
1 \text { for } R S V \text { group } \\
0 \text { for influenza group }
\end{array}\right.
$$

and

$$
T_{t}=\left\{\begin{array}{c}
1 \text { for } t \geq 2012 / 13 \\
0 \text { otherwise }
\end{array}\right.
$$

It should be noted that Eq. [1] does not involve a time trend; however, we examined various other models with a time trend [see "Model comparison" section and Supplementary file (Appendix 1)]. Similarly, we analyzed the age-specific data via five separate ordinary least square regressions. The expected incidence is described in the Supplementary file (Appendix 1).

\section{Model comparison}

In the abovementioned Eq. [1], a constant baseline and a constant causal effect were adopted, but we considered all possible patterns of causal model (i.e., a model with timedependence in either the baseline or the causal effect or with time-dependence in both the baseline and causal effect). To do so, we examined all possible combinations of time-dependence in the baseline trend and causal effect described in Eqs. [5-8] in the Supplementary file (Appendix 1). In the present study, we did not account for spatial dependence for simplicity and also because spatial heterogeneity of the causal effect was not substantially plausible in practice. Subsequently, the best model was chosen by identifying the model with the lowest Akaike information criterion (AIC) value and by verifying that the chosen model was theoretically consistent with epidemiological interpretations of the observed data.

\section{Statistical analysis}

For the descriptive and trend analyses, likelihood ratio test was employed to judge whether the proportion of clinically severe RSV infections varied over time. For this purpose, two different models (i.e., a model with and without a change point in 2012/13) were fitted to the observed virus surveillance data using a Poisson regression method. Also, we explored if the CFR and the proportion of notifications from clinics out of the total notifications changed with time. Because these are proportions, binomial models with and without change from 2012/13 season were fitted and compared to detect the trend. The causal effect $\beta$ on the incidence of RSV infection was estimated via ordinary least squares. The $95 \%$ confidence intervals (CIs) of parameters were computed by profile likelihood. All statistical analyses were conducted using JMP version 12.0.1 statistical software (SAS Institute Inc., Cary, NC, USA), and P values of $<0.05$ were considered as statistically significant.

\section{Ethical statement}

The present study used publicly available data (26). These datasets had already been fully anonymized and did not include any identity information. Thus, ethical approval and informed consent were not required for the analysis. The study was conducted in accordance with the Declaration of Helsinki (as revised in 2013).

\section{Data sharing policy}

Surveillance data for RSV infections and detections can be accessed online with the linked URL $(26,32)$, and 

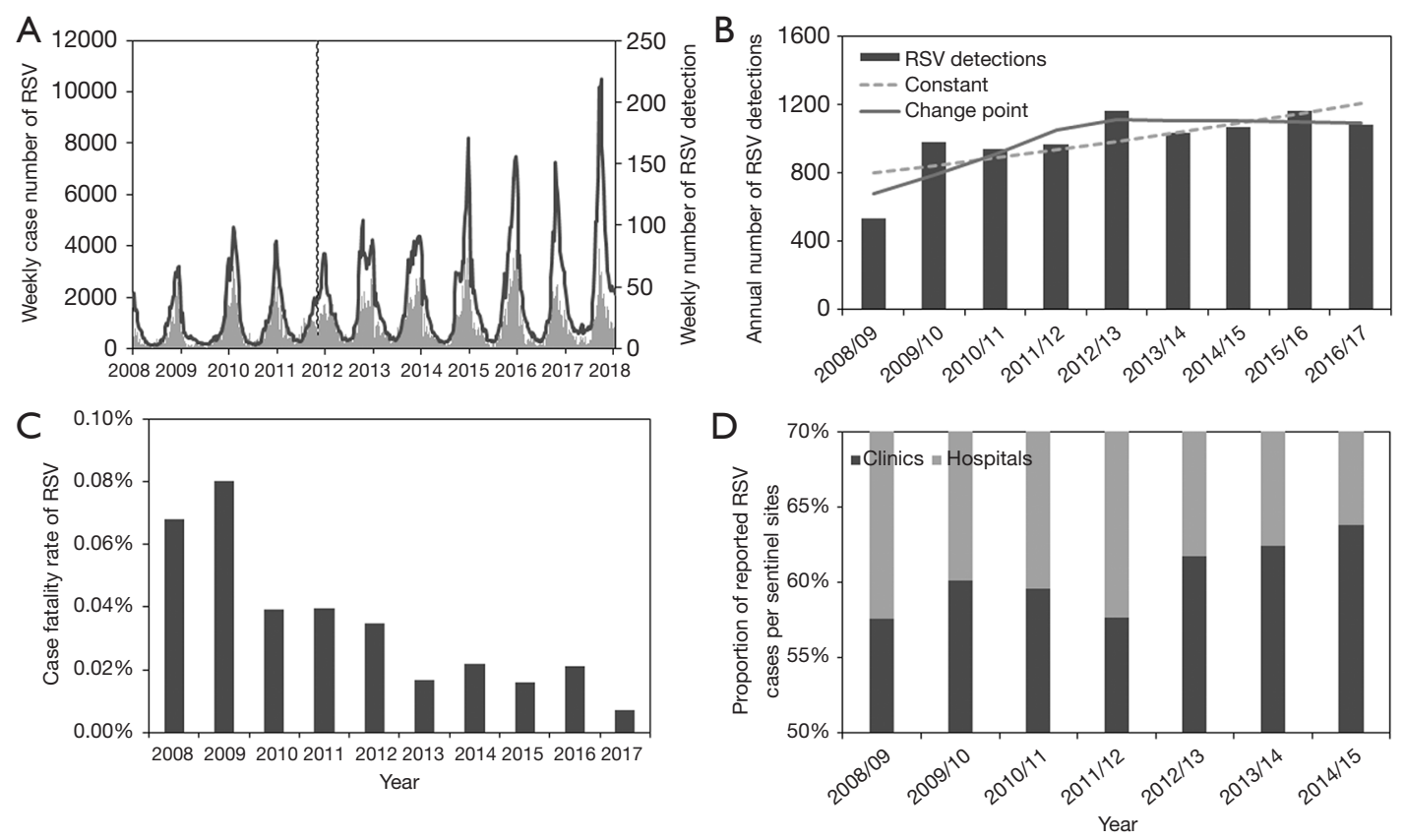

Figure 1 The incidence of RSV in Japan, 2008/09-2016/17. (A) Weekly trend of notified RSV infections (black lines) and virus detections (grey bars); (B) comparison between observed and modelled annual numbers of RSV detections. Bars show the number of RSV detection by year. Solid line and dashed line indicate the modelled number of RSV detection using Poisson regression model with and without a change point in 2012/13 season; (C) annual estimate of the CFR (i.e., the number of deaths due per notified RSV infection) of RSV infection. Deaths in 2012/13 season are mostly included as part of the 2013, and thus, the changepoint was regarded as 2013; (D) proportion of notifications by sentinel sites. The proportion of notifications from clinics is measured by dark grey bars, and light gray bars represent those from hospitals. RSV, respiratory syncytial virus; CFR, case fatality risk.

population census data are also accessible (31). A summary of the secondary datasets that were analyzed in the present study can be shared by the corresponding author upon request.

\section{Results}

To understand the temporal dynamics, the incidence of RSV infections is illustrated in Figure 1. Figure $1 \mathrm{~A}$ overlays the weekly number of reported RSV infections from the national sentinel surveillance (shown as curves) and the number of RSV detections from virus surveillance (bars). From a visual assessment of the data shown in Figure 1A, the number of notifications shows an increasing trend over time, while the number of virus detections has not clearly increased since 2012/13, which can be seen from Figure 1B. The annual trend of RSV detections was modelled using two different Poisson regression models, and a model with a change point in 2012/13 yielded a significantly better goodness-offit, compared to a constantly growing model $\left(\chi_{2}^{2}=73.2\right.$,
$\mathrm{P}<0.0001)$. In the better fitted model, even a statistically significant decrease from 2012/13 was indicated, while the model without any change point showed an increasing trend over time. Figure $1 C$ shows an estimated CFR over time. The risk of death has clearly taken a small decreasing value from 2012-2013 $\left(\chi_{1}^{2}=5.2, \mathrm{P}=0.02\right)$. Figure $1 D$ shows the proportion of notifications from clinics as a function of time. The proportion of notified RSV infections from clinics has significantly increased from 2012-2013 season $\left(\chi_{1}^{2}=1300.3, \mathrm{P}<0.0001\right)$.

The age-dependent incidences of both influenza and RSV infection from 2008/09 to 2016/17 are shown in Figure 2. Incidence data for both infections revealed that the highest estimated incidences occurred among young children, with a decreasing trend as age increased. In the case of RSV infection, a big gap was identified between children of $<10$ years old and adults aged $\geq 20$ years. The median incidences of influenza and RSV infection among those aged 0-4 years were estimated at 5,607 and 1,761 per 100,000 individuals, respectively, whereas they were estimated at 

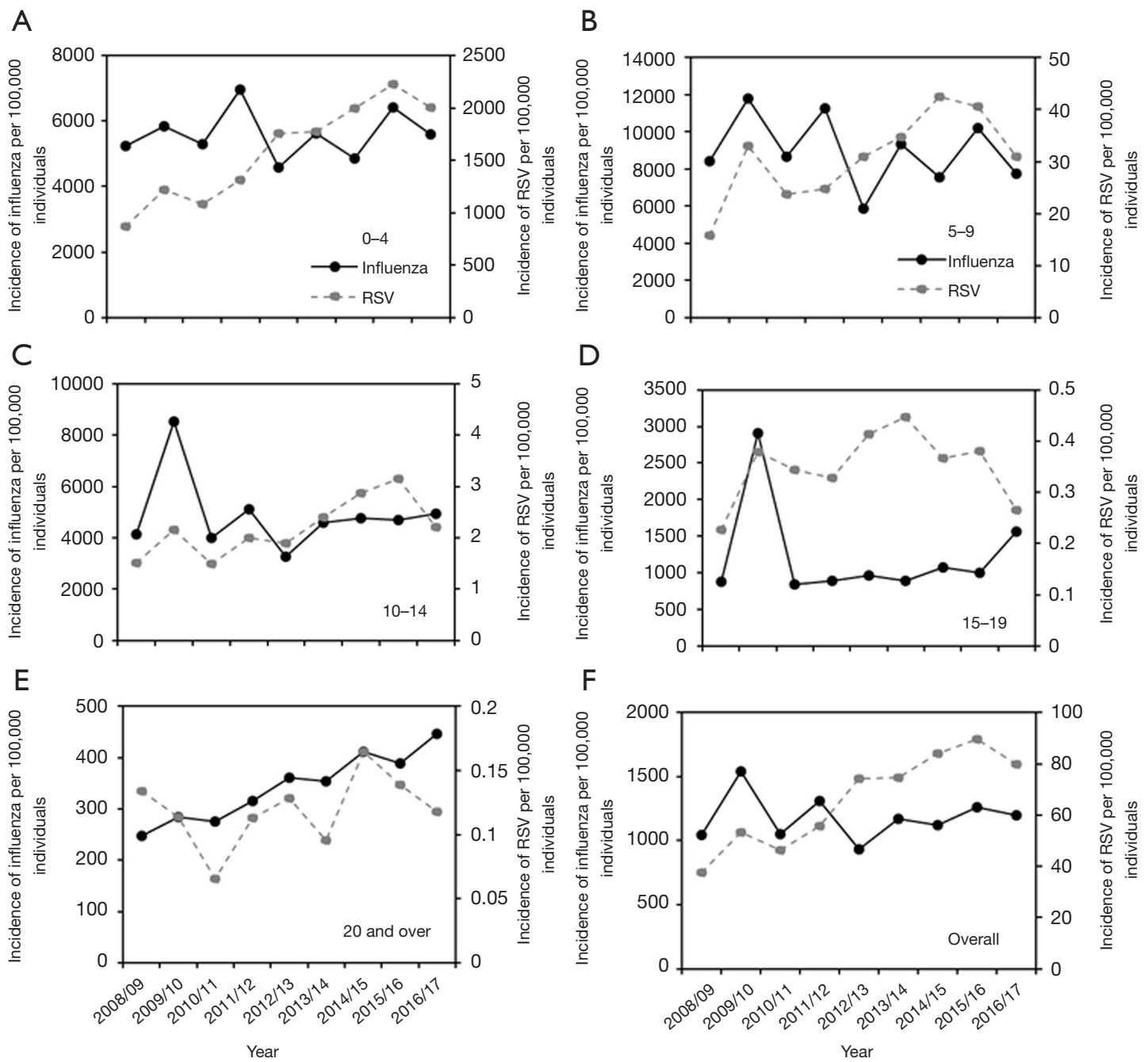

Figure 2 The incidence trends of influenza and RSV in Japan, 2008/09-2016/17. (A,B,C,D,E) Age-specific incidence trends of influenza and RSV infection based on age-specific incidence data; (F) an overall incidence trends of both infections among total population based on prefecture-specific data (i.e., incidence data by prefecture). Solid lines and dashed lines represent the observed data for influenza and RSV infection until 2016/17, respectively. RSV, respiratory syncytial virus.

354 and 0.12 per 100,000 individuals, respectively, among those aged $\geq 20$ years. Common increasing trend is seen in 2008/09 and 2009/10 for both RSV and influenza infections, which coincided with the timing of pandemic influenza A/H1N1-2009.

The estimated differences in parallel trend by age-group between the incidences of influenza and RSV infection prior to the change in national health insurance are shown in Figure 3. For all age-groups, the estimated differences in the trend slopes between the two diseases were not statistically significant, and this finding is supportive of the validity of the parallel trend assumption.
We compared four different DID models (Table S1). The AIC of each model was computed. Eq. [1] and Eq. [5] with constant baseline trends and constant causal effects yielded the lowest AIC values, for both prefecture- and agespecific incidence data. The estimates from four different models, analyzing prefecture- and age-specific incidence data are shown in Table S2.

We then employed DID Eq. [1] to capture the prefecture-specific patterns and compare the observed and estimated incidences for both influenza and RSV infections, the results of which are shown in Figure 4. Up to the 2011/12 season, the absence of a trend (i.e., a constant 


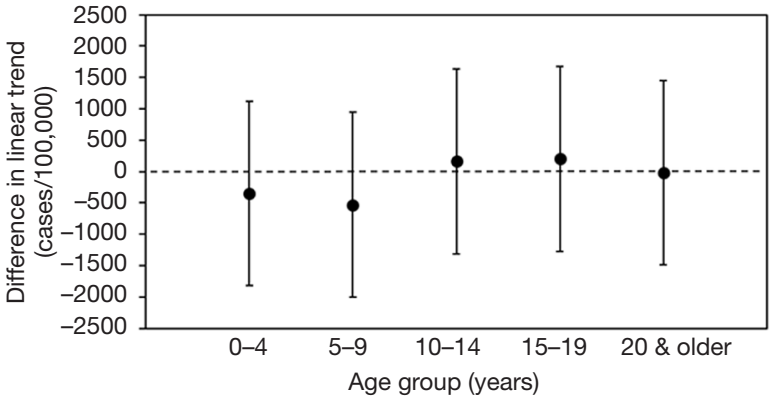

Figure 3 Difference in the linear trend slopes between influenza and RSV infection during the pre-intervention period in Japan. The estimated difference contained the value zero within the $95 \%$ CIs for each age group, demonstrating a parallel trend between influenza and RSV during the pre- intervention period (2008/092011/12). Filled circles represent expected values, and whiskers extend to upper and lower 95\% CIs. A difference at the value zero (dashed line) indicates that the assumed parallel trend is not rejected for each age group. RSV, respiratory syncytial virus; CI, confidence interval.

incidence) was seen for both infectious diseases. From the 2012/13 season onwards, the expected value of influenza incidence decreased due to the time effect for both infections, while the incidence of RSV infection abruptly increased. This phenomenon, captured by the simple DID model, is interpreted as evidence of a non-zero causal effect by the change in insurance policy to cover the cost of RSV antigen testing; on average, 162 cases (95\% CI: 85-240) of RSV infection per year were additionally diagnosed from 2012 onward (Table 1).

Table 1 shows the mean incidences of both influenza and RSV infection in pre- and post-intervention. Incidence data for both infections revealed the highest estimated incidences occurred among young children, with a decreasing trend as age increased. The mean incidence of $\mathrm{RSV}$ infection increased in post-intervention period (i.e., 2012/13-2016/17), compared to pre-intervention period (2008/09-2011/12) in every age-groups, while incidence of influenza was shown decreasing pattern in most age-groups.

In addition, the estimated age-specific unadjusted and adjusted causal parameters $\beta$ are also shown in Table 1; its values indicate that the causal effect of RSV testing on the increase in yearly RSV infection incidence adjusted with time-dependent effect $(\delta)$ and group-dependent effect $(\gamma)$ was greatest among children aged 5-9 years [an additional 1,912 cases (95\% CI: 418-3,406)] (Figure 5). Except for
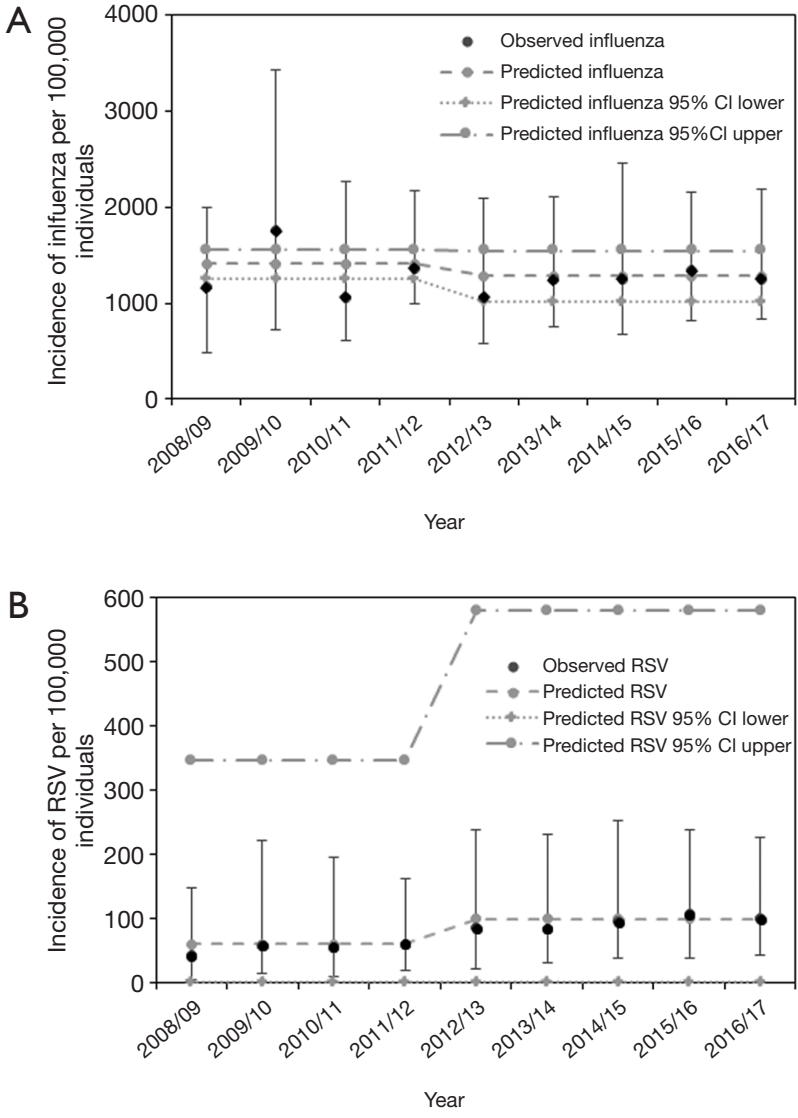

Figure 4 Comparison of observed and predicted incidence from the analysis of prefectural data. (A,B) The predicted incidence per 100,000 individuals for influenza (A) and RSV (B) infection, calculated using prefectural data, are shown as grey points. Black points represent the median of the observed incidence, and error bars indicate the minimum and maximum of the observed values among the 47 prefectures in Japan. Dash-dotted lines and dotted lines represent the upper and lower bound, respectively, of the 95\% CI obtained from samples of estimated parameters generated via bootstrapping 1,000 times. RSV, respiratory syncytial virus; CI, confidence interval.

children aged 5-9 years old, the causal effects were not statistically significant, and the precision of the causal effect was low with broad CIs.

Figure 6 examines the overall good agreement between the observed and expected age-specific incidence data. A statistically significant decline in the influenza incidence and a growing pattern of the RSV incidence were identified from 2012/13 onward among those aged 5-9 years, using the age-specific model. 
Table 1 Incidence of influenza and RSV infection by age in pre- and post-intervention and estimates of the causal effect parameter ( $\beta$ ) of the national health insurance policy change in RSV antigen detection test funding by age in Japan

\begin{tabular}{|c|c|c|c|c|c|c|}
\hline $\begin{array}{l}\text { Age } \\
\text { (years) }\end{array}$ & \multicolumn{2}{|c|}{ Mean incidence of RSV } & \multicolumn{2}{|c|}{ Mean incidence of influenza } & $\begin{array}{l}\text { Unadjusted estimates } \\
\qquad[95 \% \mathrm{Cl}]\end{array}$ & $\begin{array}{c}\text { Adjusted estimates } \\
{[95 \% \mathrm{Cl}]}\end{array}$ \\
\hline $0-4$ & 1,121 & 1,953 & 5,835 & 5,412 & $-2,269[-4,622,83]$ & $1,255[-219,2,749]$ \\
\hline $5-9$ & 24 & 36 & 10,048 & 8,147 & $-6,197[-8,549,-3,844]$ & $1,912[418,3,406]$ \\
\hline $10-14$ & 2 & 3 & 5,452 & 4,457 & $-3,390[-5,742,-1,037]$ & $995[-499,2,489]$ \\
\hline$\geq 20$ & 0.11 & 0.13 & 281 & 393 & $-237[-2,590,2,115]$ & $-112[-1,606,1,382]$ \\
\hline Overall & 61 & 99 & 1,408 & 1,284 & $-847[-934,-759]$ & $162[85,240]$ \\
\hline
\end{tabular}

Mean incidence (i.e., reported cases) per 100,000 individuals for both infections in pre- and post- intervention are shown. The unadjusted and adjusted estimates ( $\beta$ ) should be interpreted as the increase in yearly incidence of RSV infection per 100,000 individuals due to the change in the national insurance policy. Estimates were adjusted with time-dependent effect $(\delta)$ and group-dependent effect $(\gamma)$. 95\% Cls of each estimates were calculated through profile likelihood. All estimates were derived from an age-dependent model, except for the last row, which presents estimated parameters from prefecture-dependent model, interpreted as the effect of the total population. RSV, respiratory syncytial virus; $\mathrm{Cl}$, confidence interval.

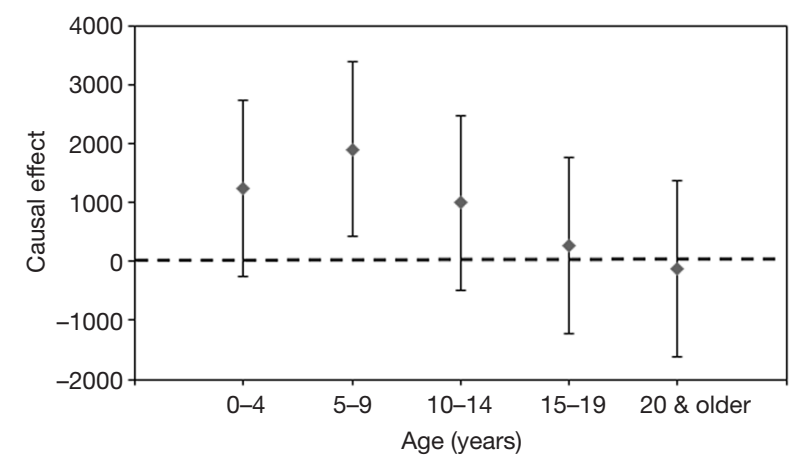

Figure 5 Age-specific causal effect of the national health insurance policy change in funding RSV antigen testing. The age-specific causal effects of the expansion of the national health insurance coverage for RSV antigen testing are shown as grey points, and error bars represent the $95 \%$ CI of the age-specific causal effect. RSV, respiratory syncytial virus; CI, confidence interval.

\section{Discussion}

The present study aimed to estimate the causal impact on the incidence of RSV infection of a change in the national health insurance policy to financially cover the cost of RSV antigen detection testing. The annual number of notified RSV cases has shown an increasing trend, while the number of RSV detections from virus surveillance has shown a significant decreasing trend since 2012/13. CFR and the proportion of notifications from clinics were indicative of diluted severity of RSV infections since 2012/13, implying that perhaps clinically mild cases have been notified more than before since 2012/13. Employing a DID model, a significant causal effect was revealed among those aged 5-9 years, with the effect of 1,912 additional cases per 100,000 persons, from the 2012/13 season onward. Overall, a statistically significant effect of the policy was observed as 162 cases above the baseline level and that this effect was primarily driven by a change in RSV incidence among 5-9 years old. Our simple Eq. [1] and Eq. [5] successfully captured the observed increasing patterns of RSV infection incidence in Japan, demonstrating that the increase could be largely explainable as induced by the change in the national health insurance policy. To our knowledge, the present study is the first to have explicitly estimated the causal effect of such an insurance policy change on infectious disease incidence using a DID model.

As an important contribution of the present study, we have confirmed the existence of an increased incidence of RSV infection following the introduction of RDT kit coverage by insurance. That is, the ongoing excess of RSV infection in recent years compared with earlier times before 2012/13 must not be immediately interpreted as a real increase in the incidence of RSV infections. Even descriptive and trend analyses were indicative that an abrupt increase in RSV notifications from 2012/13 were not caused by actual increase of infections over time. Rather, we must always discount an average of 162 additional cases from the datasets generated in 2012/13 and later, due to the change in insurance policy at this time. The actual identifiable 


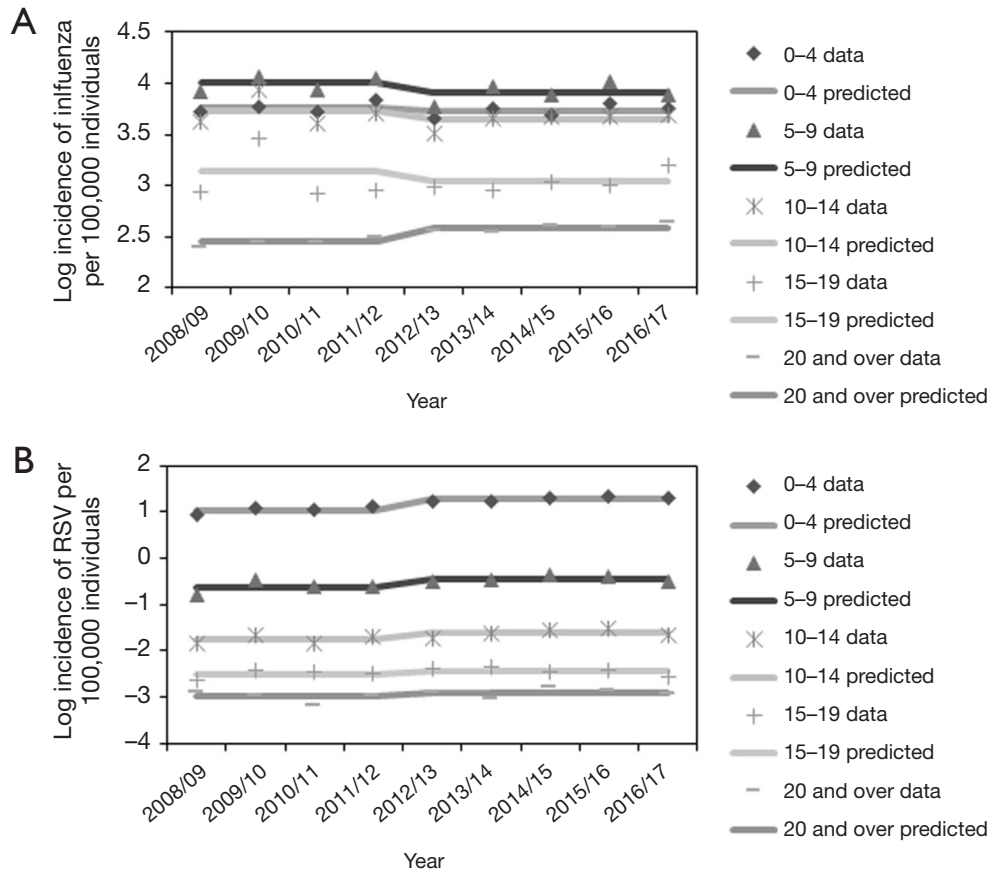

Figure 6 Comparison of observed and predicted age-specific incidences. (A,B) Age-specific log incidence of influenza (A) and RSV (B) infection, from the analysis of age-specific data. As most reported influenza and RSV infection cases occur in those aged < 9 years, a logarithm scale is used in both figures to allow visibility of the fitness in every age-group. Each line represents the predicted incidence, while each sign represents the observed incidence data. RSV, respiratory syncytial virus.

difference in the observed RSV infection incidence data was smaller than 162 cases because other parameters, including $\gamma$ and $\delta$, also adjusted the difference in incidence between influenza and RSV infection, and the actual increase induced by the funding coverage of bedside antigen testing was about 43 additional cases. By decomposing the timedependent changes in RSV incidence, the present study has quantified the extent of this increase. If far more than 43 cases per year are identified in the absence of any other breakthroughs, that would lead us to believe that the incidence has naturally increased and would serve as a call for the exploration of other causes for the increase. The present study has not identified any signature of natural increase in the incidence of RSV infection through 2016/17.

The age-specific effect detected by this study was very large in children aged 5-9 years old. Although the incidence of RSV infection among 5-9 years old is very small, with 31 cases per 100,000 individuals as the median from 2008/09-2016/17, the causal effect of the change in policy was visible through the DID model. From the start season of policy change (i.e., 2012/13), incidence of both RSV infection and influenza among those aged
0-4 years old have abruptly increased. However, the incidence of influenza among group of children aged 5-9 years was constant, while the incidence of RSV infection has shown increasing pattern. Therefore, given that the national health insurance extended its coverage to all outpatients for whom palivizumab is prescribed and the majority of RSV infection cases is those aged below 10 years old, this causal effect among the 5-9 years old age-group is plausible. However, the group of children aged 5-9 years old also involved a large estimate of the time-dependent $\gamma$ decrease in both influenza and RSV infection; thus, the actual increase in RSV incidence from 2012/13 in this group remained very small (i.e., less than 1 case per 100,000). The diminished actual effect can, of course, be attributed to the use of influenza as a control, but its use was at least validated through the results of linear trend analyses (Figure 3). The use of influenza as the control disease for our study may be perceived as an unsupported assumption, but, as we have shown, correlations between influenza and RSV infection were diminished from 2012/13 in all groups, except for those aged $\geq 15$ years, who are far less affected by RSV infection compared with younger 
children. Moreover, the decomposition of the role of Eq. [1] is very transparent, and, as we show in Table 1, each parameter estimate was made explicitly interpretable.

The rise of incidence of RSV infection was mostly driven by those aged 0-4 and 5-9 years old and statistically significant causal effect was observed in only among the 5-9-year-old age-group. An increase of RSV infection among $0-4$ years old might be not related with the change of funding coverage of RSV bedside antigen testing, while the possible increase among $0-4$ years old must be continuously monitored, considering the clinical impact among infants aged from $0-2$ years. Nevertheless, the significant increase induced by policy change was observed among aged 5-9 years old and total population and furthermore, the CFR of RSV infection shows decreasing trend over time (Figure 1C) and the notified RSV infections from clinics have increased than from hospitals (Figure 1D), which can be perceived as an increase in testing frequency. Thus, this study identified that the incidence of RSV infection has increased due to providing RSV antigen testing with financial support, while a natural increase in incidence of RSV infection also cannot be fully rejected except those aged 5-9 years old.

Several limitations of our study must be described. First, we examined only sentinel surveillance data. Thus, the incidences of influenza and RSV infection used in our models represent only a portion of the actual incidence of infection and were biased by medical-seeking behaviors. Nevertheless, we believe that the time trend of prefectureand age-specific incidences of both influenza and RSV infection were sufficiently captured, and crude timedependent patterns of the reported fraction of infections were adequately described. Second, as the simplest DID model was selected following a comparison of AIC values (Table S1), and yearly fluctuations in influenza incidence were not fully captured, e.g., influenza A (H1N1-2009) pandemic, the simplest model had to regard that variation as the measurement of error. Third, although RDT is performed in Japan to diagnose influenza for the majority of both in- and outpatients (30), as clinical symptoms of both infections are hardly distinguishable from one another, there remains the possibility that RSV infection cases partially (but very rarely) might be included in the datasets of "influenza" reporting (33-35). Fourth, similar scaling between the case and control groups would be ideally required (36), but the scale for influenza infection is bigger than that of RSV infection. However, among childhood upper respiratory diseases with similar etiology and epidemiology that lack notable breakthroughs in prevention and treatment during the study timeframe, there is no more suitable disease than influenza to use as a control disease for RSV. Fifth, although the mortality burden of both influenza and RSV infection are expected to be highest among elderly, unfortunately, the diagnosis using RDT is devised only in pediatric settings and the detailed age breakdown of RSV incidence among adults is unknown. An explicit estimation of the mortality burden of RSV requires additional effort of exploration in this particular age group. Finally, no control groups other than influenza were considered because there were no infectious diseases with sentinel surveillance that could be assumed as holding a parallel assumption and better satisfied other conditions to become a control. By identifying alternative control groups, we could adjust ourselves to validate the causal effect estimate.

\section{Conclusions}

While the present study faces these technical limitations, this study has suggested that recently increasing incidence of RSV infection is not caused by actual increase in the number of infections, and quantified the impact of the change in national health insurance policy on the RSV infection incidence. Due to providing RSV antigen testing with financial support beginning in October 2011, the incidence of RSV infection increased by about 162 cases per 100,000 individuals per year, and therefore, the recent increase should not immediately be perceived as indicative of a natural increase in the incidence of RSV infections. Caution should be practiced for the interpretation on similar trend of surveillance data with the bias due to the systematical changes in diagnosis. Furthermore, better identification of RSV infection through widespread RSV antigen testing might help avoid an under-ascertainment of any further increases in the incidence of RSV infection.

\section{Acknowledgments}

We thank Katie Oakley, PhD, from Edanz Group (www. edanzediting.com/ac) for editing a draft of this manuscript. Funding: HL's study was supported by the Japan Society for the Promotion of Science (JSPS) Program for Advancing Strategic International Networks to Accelerate the Circulation of Talented Researchers. HN received funding support from the Japan Agency for Medical Research and Development (AMED, grant number JP18fk0108050), JSPS KAKENHI (Grant Numbers 16KT0130, 16K15356, 
$17 \mathrm{H} 04701,17 \mathrm{H} 05808$, and $18 \mathrm{H} 04895)$, Inamori Foundation, and the Japan Science and Technology Agency (JST) CREST program (JPMJCR1413). SMJ thanks The Ministry of Education, Culture, Sports, Science, and Technology (MEXT), Japan for its scholarship program.

\section{Footnote}

Reporting Checklist: The authors have completed the MDAR checklist. Available at http://dx.doi.org/10.21037/atm-20423

Conflicts of Interest: All authors have completed the ICMJE uniform disclosure form (available at http://dx.doi. org/10.21037/atm-20-423). The authors have no conflicts of interest to declare.

Ethical Statement: The authors confirm that the authors are accountable for all aspects of the work in ensuring that questions related to the accuracy or integrity of any part of the work are appropriately investigated and resolved. The present study used publicly available data, which had already been fully anonymized and did not include any identity information. Thus, ethical approval and informed consent were not required for the analysis. The study was conducted in accordance with the Declaration of Helsinki (as revised in 2013).

Open Access Statement: This is an Open Access article distributed in accordance with the Creative Commons Attribution-NonCommercial-NoDerivs 4.0 International License (CC BY-NC-ND 4.0), which permits the noncommercial replication and distribution of the article with the strict proviso that no changes or edits are made and the original work is properly cited (including links to both the formal publication through the relevant DOI and the license). See: https://creativecommons.org/licenses/by-nc-nd/4.0/.

\section{References}

1. Sruamsiri R, Kubo H, Mahlich J. Hospitalization costs and length of stay of Japanese children with respiratory syncytial virus: a structural equation modeling approach. Medicine (Baltimore) 2018;97:e11491.

2. Howard TS, Hoffman LH, Stang PE, et al. Respiratory syncytial virus pneumonia in the hospital setting: length of stay, charges, and mortality. J Pediatr 2000;137:227-32.

3. Simoes EA, Carbonell-Estrany X. Impact of severe disease caused by respiratory syncytial virus in children living in developed countries. Pediatr Infect Dis J 2003;22:S13-8; discussion S18-20.

4. Committee on Infectious Diseases. From the American Academy of Pediatrics: Policy statements--Modified recommendations for use of palivizumab for prevention of respiratory syncytial virus infections. Pediatrics 2009;124:1694-701.

5. Jackson DJ, Gangnon RE, Evans MD, et al. Wheezing rhinovirus illnesses in early life predict asthma development in high-risk children. Am J Respir Crit Care Med 2008;178:667-72.

6. Weber A, Weber M, Milligan P. Modeling epidemics caused by respiratory syncytial virus (RSV). Math Biosci 2001;172:95-113.

7. Lloyd PC, May L, Hoffman D, et al. The effect of birth month on the risk of respiratory syncytial virus hospitalization in the first year of life in the United States. Pediatr Infect Dis J 2014;33:e135-40.

8. Nair H, Nokes DJ, Gessner BD, et al. Global burden of acute lower respiratory infections due to respiratory syncytial virus in young children: a systematic review and meta-analysis. Lancet 2010;375:1545-55.

9. Tatochenko V, Uchaikin V, Gorelov A, et al. Epidemiology of respiratory syncytial virus in children $\leq 2$ years of age hospitalized with lower respiratory tract infections in the Russian Federation: a prospective, multicenter study. Clin Epidemiol 2010;2:221-7.

10. Ren L, Gonzalez R, Wang Z, et al. Prevalence of human respiratory viruses in adults with acute respiratory tract infections in Beijing, 2005-2007. Clin Microbiol Infect 2009;15:1146-53.

11. Chan PK, Sung RY, Fung KS, et al. Epidemiology of respiratory syncytial virus infection among paediatric patients in Hong Kong: seasonality and disease impact. Epidemiol Infect 1999;123:257-62.

12. Welliver R. The relationship of meteorological conditions to the epidemic activity of respiratory syncytial virus. Paediatr Respir Rev 2009;10 Suppl 1:6-8.

13. Law BJ, Carbonell-Estrany X, Simoes EA. An update on respiratory syncytial virus epidemiology: a developed country perspective. Respir Med 2002;96 Suppl B:S1-7 .

14. Welliver RC. Review of epidemiology and clinical risk factors for severe respiratory syncytial virus (RSV) infection. J Pediatr 2003;143:S112-7.

15. Wang D, Cummins C, Bayliss S, et al. Immunoprophylaxis against respiratory syncytial virus (RSV) with palivizumab in children: a systematic review and economic evaluation. 
Health Technol Assess 2008;12:iii, ix-x, 1-86.

16. Bloom-Feshbach $\mathrm{K}$, Alonso WJ, Charu V, et al. Latitudinal variations in seasonal activity of influenza and respiratory syncytial virus (RSV): a global comparative review. PLoS One 2013;8:e54445.

17. Taniguchi K, Hashimoto S, Kawado M, et al. Overview of infectious disease surveillance system in Japan, 1999-2005. J Epidemiol 2007;17 Suppl:S3-13.

18. National Institute of Infectious Diseases. Respiratory syncytial virus infection, as of May 2014. 2014. Available online: https://www.niid.go.jp/niid/en/iasr-vol33-e/865iasr/4769-tpc412.html

19. Woo PC, Chiu SS, Seto WH, et al. Cost-effectiveness of rapid diagnosis of viral respiratory tract infections in pediatric patients. J Clin Microbiol 1997;35:1579-81.

20. Takeuchi Y, Nakata S, Asamura S, et al. A new rapid diagnostic kit for respiratory syncytial virus infections evaluation. Kansenshogaku Zasshi 2010;84:309-12.

21. Kanou K, Arima Y, Kinoshita H, et al. Respiratory syncytial virus surveillance system in Japan: assessment of recent trends, 2008-2015. Jpn J Infect Dis 2018;71:250-5.

22. Shobugawa Y, Saito R, Sano Y, et al. Emerging genotypes of human respiratory syncytial virus subgroup A among patients in Japan. J Clin Microbiol 2009;47:2475-82.

23. Stuart EA, Huskamp HA, Duckworth K, et al. Using propensity scores in difference-in-differences models to estimate the effects of a policy change. Health Serv Outcomes Res Methodol 2014;14:166-82.

24. Ryan AM, Burgess JF Jr, Dimick JB. Why we should not be indifferent to specification choices for difference-indifferences. Health Serv Res 2015;50:1211-35.

25. Dimick JB, Ryan AM. Methods for evaluating changes in health care policy: the difference-in-differences approach. JAMA 2014;312:2401-2.

26. National Institute of Infectious Diseases. Infectious

Cite this article as: Jung SM, Lee H, Yang Y, Nishiura H. Quantifying the causal impact of funding bedside antigen testing on the incidence of respiratory syncytial virus infection in Japan: a difference-in-differences study. Ann Transl Med 2020;8(21):1441. doi: 10.21037/atm-20-423
Disease Weekly Report (IDWR). Available online: https:// www.niid.go.jp/niid/en/idwr-e.html

27. Popow-Kraupp T, Aberle JH. Diagnosis of respiratory syncytial virus infection. Open Microbiol J 2011;5:128-34.

28. Kaneko M, Watanabe J, Kuwahara M, et al. Impact of respiratory syncytial virus infection as a cause of lower respiratory tract infection in children younger than 3 years of age in Japan. J Infect 2002;44:240-3.

29. Mizumoto K, Yamamoto T, Nishiura H. Age-dependent estimates of the epidemiological impact of pandemic influenza (H1N1-2009) in Japan. Comput Math Methods Med 2013;2013:637064.

30. Sakai-Tagawa Y, Ozawa M, Tamura D, et al. Sensitivity of influenza rapid diagnostic tests to H5N1 and 2009 pandemic H1N1 viruses. J Clin Microbiol 2010;48:2872-7.

31. Statistics Bureau of Japan. Population Estimates. Available online: https://www.e-stat.go.jp/stat-search/files?page $=1 \& \mathrm{t}$ oukei $=00200524 \&$ tstat $=000000090001$

32. National Institute of Infectious Diseases. Infectious Agents Surveillance Report. Available online: https://www.niid. go.jp/niid/ja/iasr.html

33. Falsey AR, Hennessey PA, Formica MA, et al. Respiratory syncytial virus infection in elderly and high-risk adults. $\mathrm{N}$ Engl J Med 2005;352:1749-59.

34. Fleming DM, Pannell RS, Elliot AJ, et al. Respiratory illness associated with influenza and respiratory syncytial virus infection. Arch Dis Child 2005;90:741-6.

35. Wallace LA, Collins TC, Douglas JD, et al. Virological surveillance of influenza-like illness in the community using PCR and serology. J Clin Virol 2004;31:40-5.

36. Jung SM, Lee H, Nishiura $H$. The impact of pneumococcal vaccination on pneumonia mortality among the elderly in Japan: a difference-in-difference study. PeerJ 2018;6:e6085. 